The International Journal Of Engineering And Science (IJES)

|| Volume || 5 || Issue || 11 || Pages || PP 01-07 || 2016 ||

ISSN (e): $2319-1813$ ISSN (p): $2319-1805$

\title{
Recognition Approach of Visual Images Based on Standards and Training
}

\author{
Batyrkanov Jenish Isakunovich ${ }^{1}$, Kudakeeva Gulida Madanbekovna ${ }^{2}$, \\ Subankulova Jyldyz Janybekovna ${ }^{3}$ \\ ${ }^{I}$ D.Sc.(Engineering), Professor, Kyrgyz State Technical University after name of I.Razzakov,66 Mir Ave. \\ Bishkek, 720044, Kyrgyz Republic \\ ${ }^{2}$ post-graduate student of “Automatic control”, Kyrgyz. State Technical University after name of I.Razzako, 66 \\ Mir Ave., Bishkek, 720044, Kyrgyz Republic \\ ${ }^{3}$ researchers of "Automatic control”, Kyrgyz State Technical University after name of I.Razzako, 66 Mir Ave., \\ Bishkek, 720044, Kyrgyz Republic
}

\begin{abstract}
There are two variants of a pattern recognition algorithm. In the first variant recognition algorithm uses the "proximity principle" and finds the minimum norm of the difference of the presented numerical matrix image with a numerical matrix of reference images. Recognition algorithm uses the "proximity principle" and finds the minimum norm of the difference between presented numerical matrix for image with a numerical matrix of reference model. Found the minimum norm is the key to classification. In the second embodiment computes the dot product of a vector image on target vectors. The maximum inner product and determines the desired class. Key words: image, object recognition, classification, training, learning procedure, reference images.
\end{abstract}

$\longrightarrow$ Date of Accepted: 22 November 2016

\section{INTRODUCTION}

Modern automation systems, communications, computer networks, robots are increasingly deeper and apply different types of recognition systems. This is primarily industrial robots equipped with different touchsensitive devices are computer scanners written texts and audio information is a fingerprint recognition system, recognition of the iris, recognition of oil and gas, and many others.

Today, a very large number of applications relate to the problems of recognition of visual images. Thus under way understood a concrete manifestation of the object being studied.

Image recognition (visual images) - is a special case of pattern recognition, which signals are recognizable image, the resulting design of real-world objects on sensitive systems. Recognition of visual images is one of the most important cases for the practice of the general problem of pattern recognition. Recognition Tasks of visual images is to provide methods and devices allowing to automatically classify different images. Images can be represented on paper, photographic film or other materials.

There are various approaches and algorithms for recognition of visual images: an approach based on the calculation of estimates; approach based on analogy-based approach compared with the standards and others.

Based on the above algorithms developed various software packages: Fine Reader; From Reader; ABBY Fine Reader 4.0.

The above software packages and decide effective only for a limited class of problems of recognition, such as recognition of printed texts, uppercase characters.

In connection with the above, the development of new approaches and algorithms of recognition of visual images is - an urgent problem.

The essence of the following proposed approach to recognition is to use an approach using standards and training procedures. The structure recognizes the device shown in Figure 1. 


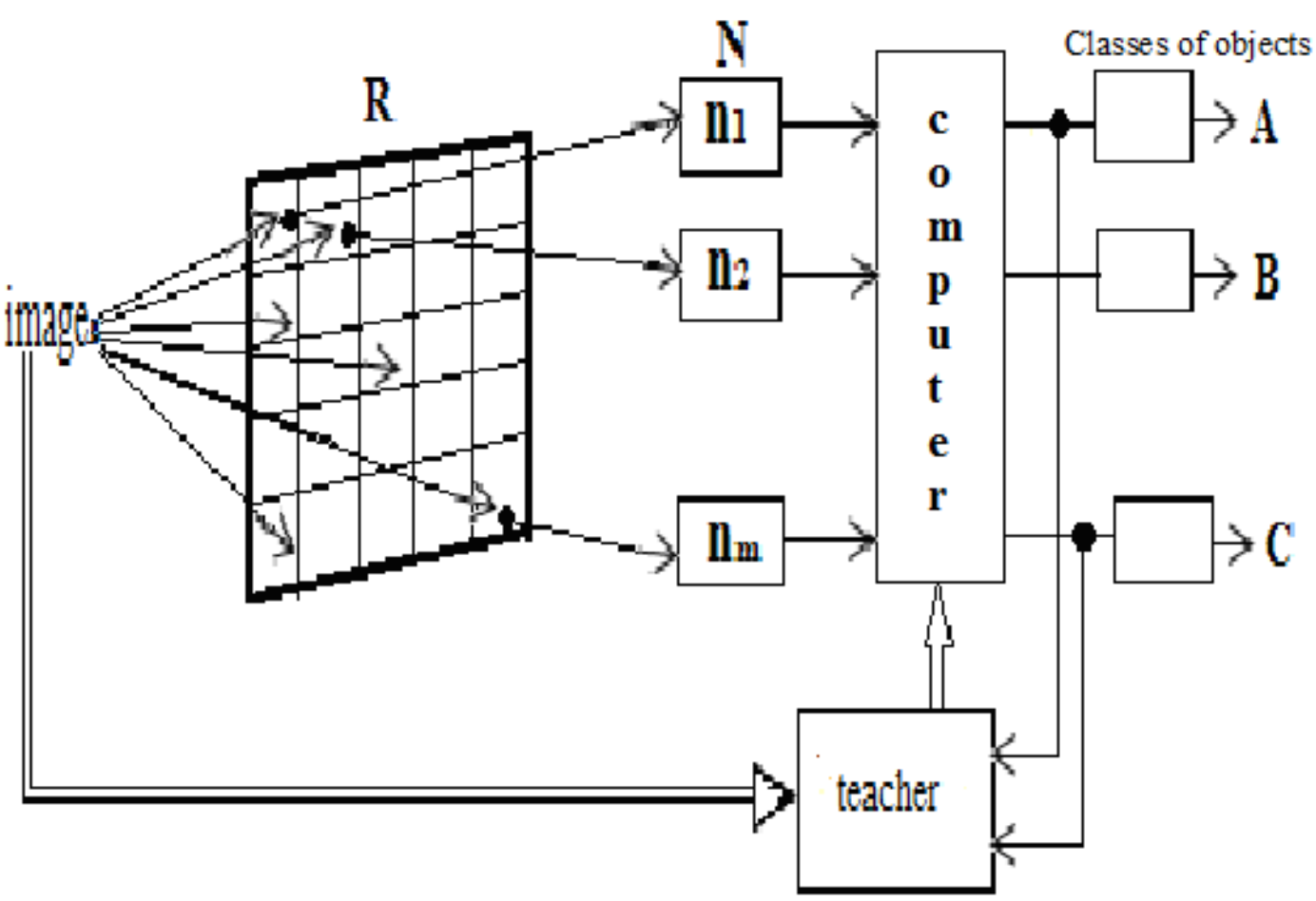

Fig.1. The structure of the recognizing device

The figure marked: R-receptor matrix; N-threshold elements; A, B, C - class facilities.

The threshold number of layer $\mathrm{N}$ elements equals the threshold number of cells the receptor matrix $\mathrm{R}$, wherein each cell of the matrix is connected, only one threshold element.

\section{The learning procedure}

The learning procedure is as follows. a certain amount of "A" of the first object images sequentially supplied to receptor matrix. Upon presentation of a specific image $\mathrm{A}^{1}$, activated by specific cell receptor matrix. Activation of cells of the matrix, can be represented as a specific numerical matrix activation, the size of numerical matrix determined by the size of the receptor matrix. We have a numerical matrix type

$$
A^{1}=\left(\begin{array}{lll}
a_{11}^{1} & a_{12}^{1} & a_{1 \mathrm{~N}}^{1} \\
a_{21}^{1} & a_{22}^{1} & a_{2 \mathrm{~N}} \\
a_{M 1}^{1} & a_{M 2}^{1} & a_{M N}^{1}
\end{array}\right),
$$

Where $a_{i j}^{1}=\left\{\begin{array}{l}0, \\ 1 .\end{array}\right.$

Further, the object $\mathrm{A}^{2}$ presented to the image of «A» and so on until the $\mathrm{A}^{\mathrm{L}}$ last possible image of the object «A». All this set of images $A^{1}, A^{2}, \ldots A^{L}$ of the object «A» is written with the help of the teacher in the computer memory as a reference image database object «A». All of the above procedure is repeated for all the objects $\mathrm{B}, \ldots, \mathrm{C}$. As a result, your computer creates a database master image data objects A, B, C ...
$A=\left\{A^{1}, A^{2}, \ldots, A^{L}\right\}$
$\mathrm{B}=\left\{\mathrm{B}^{1}, \mathrm{~B}^{2}, \ldots, \mathrm{B}^{\mathrm{P}}\right\}$
$\mathrm{C}=\left\{\mathrm{C}^{1}, \mathrm{C}^{2}, \ldots, \mathrm{C}^{\mathrm{M}}\right\}$

After the training phase begins recognition mode. 


\section{RECOGNITION ALGORITHMS}

There are two variants of the recognition algorithm.

\section{1-embodiment of the recognition algorithm}

The classification procedure, ie classification of the presented image of this or that objects are as follows. Upon presentation of the receptor matrix, for example, " $\mathrm{X}$ " of the image based on the threshold layer is formed in the computer numerical image in the form of a matrix

$$
X=\left(\begin{array}{lll}
X_{11} & X_{12} & X_{1 N} \\
X_{21} & X_{22} & X_{2 N} \\
X_{M 1} & X_{M 2} & X_{M N}
\end{array}\right),
$$

To include the image of the "X" to a particular class of objects $\mathrm{A}, \mathrm{B}, \mathrm{C} \ldots$, carried out the procedure of sequential calculation " $\mathrm{X}$ " standards on the differences of the matrix with all the matrices $\mathrm{A}^{1}{ }_{v} \mathrm{~A}^{2}, \ldots, \mathrm{A}^{\mathrm{L}} \mathrm{B}^{1}{ }_{v, \ldots}, \mathrm{C}_{,}{ }^{1} \ldots \mathrm{C}^{\mathrm{M}}$

Which are stored in the reference images of objects A, B, C ... is convenient to calculate the rate of a Euclidean norm

$$
\left\|\mathrm{X}-\mathrm{A}^{\mathrm{K}}\right\|=\left(\sum_{\mathrm{i}} \sum_{\mathrm{j}}\left(\mathrm{X}_{\mathrm{ij}}-\mathrm{a}_{\mathrm{ij}}^{\mathrm{K}}\right)^{2}\right)^{1 / 2}
$$

Among this set there is a minimum standard, even if, for example, the minimum rate differences will be for the image $B^{S}$

$$
\left\|X-B^{S}\right\|=\left(\sum_{i} \sum_{j}\left(X_{i j}-B_{i j}^{S}\right)^{2}\right)^{1 / 2}
$$

Then the computer activates the output "B" of the output layer, this shows that the presented image of the "X" refers to the object "B".

The described classification algorithm (recognition) operates not only in cases when the matrix "X" matches one of $\mathrm{A}^{1}, \ldots, \mathrm{C}^{\mathrm{M}}$, the work and in cases where " $\mathrm{X}$ " does not coincide with any one of them. But here, according to the "proximity principle", the classification is successful. The essence of the "principle of proximity" in recognition of the theory lies in the fact that the images of the same object in the feature space are located close to each other.

\section{2-embodiment of the recognition algorithm}

After implementation of the computer image as a numerical " $\mathrm{X}$ " of the matrix equation (3). Further, all the matrices $X, A^{1} \quad A^{2}, \ldots, C^{M}$ are converted to vector form as

$$
\overline{\mathrm{X}}=\left(\mathrm{X}_{11}, \mathrm{X}_{12} \ldots \mathrm{X}_{1 \mathrm{~N}}, \mathrm{X}_{21}, \ldots, \mathrm{X}_{2 \mathrm{~N}}, \ldots \mathrm{X}_{\mathrm{M} 1}, \ldots \mathrm{X}_{\mathrm{MN}}\right)^{\mathrm{T}} \text {, }
$$

Next computes the scalar product $\overline{\mathrm{X}}_{\text {with all vectors }} \overline{\mathrm{A}^{1}}, \ldots, \overline{\mathrm{C}^{\mathrm{M}}}$

The scalar product of the vector image $\overline{\mathrm{X}}$ to vector templates, $\overline{\mathrm{A}^{1}}, \ldots, \overline{\mathrm{C}^{\mathbb{M}}}$ characterized by their similarity, with the image of " $\mathrm{X}$ " refers to the class standards, for which the scalar product is given maximum value.

Consider the example of a specific target recognition by photographs (photographs).

Consider the algorithm of the program in $\mathrm{C} \#$.

At the beginning of the launch of the program interface appears, which is the main menu.

On the window can be seen a blank image, letting us know that the program has not yet been activated.

The main menu contains two options:

1. Recognition.

2. Education. 
It looks like this, see. Fig.2.

As before, how to recognize the need to teach the program, click on the Education button.

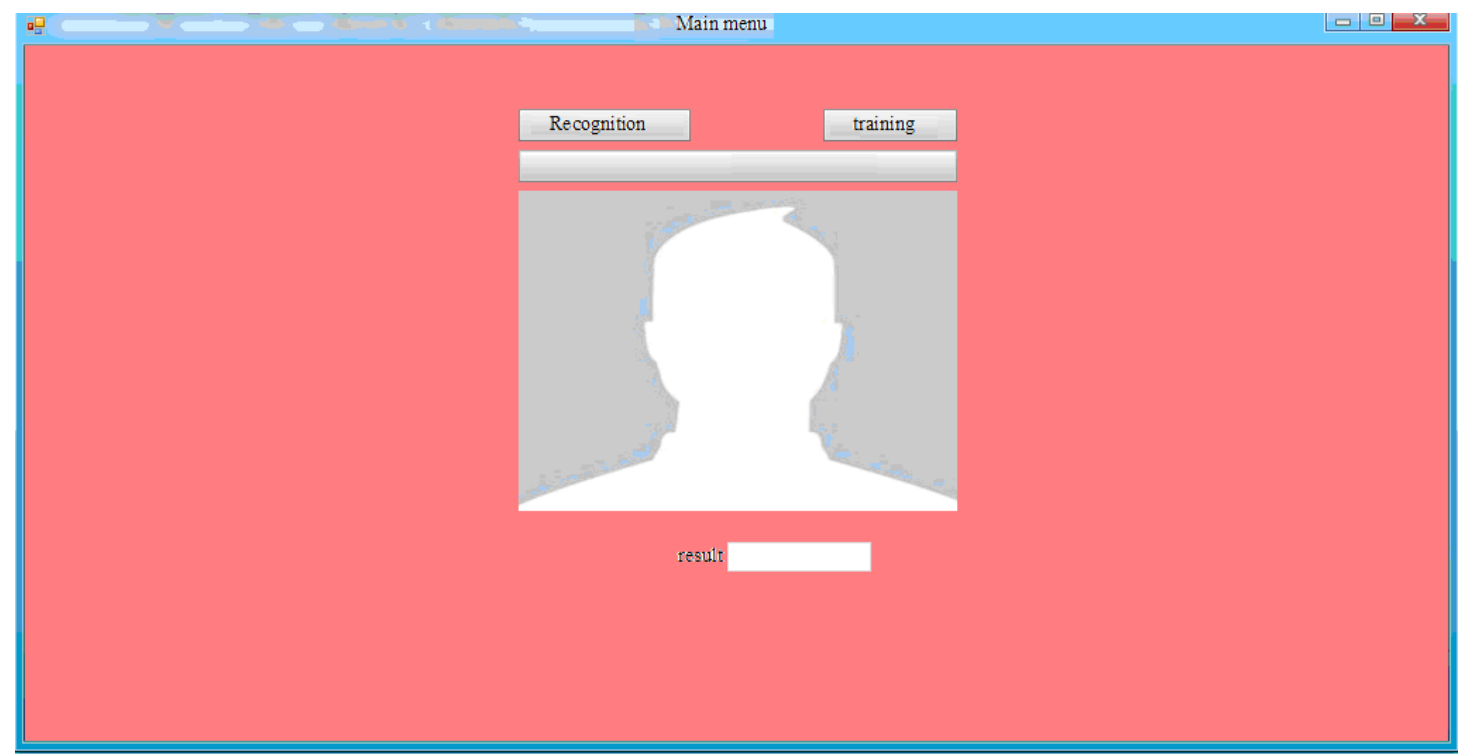

Fig.2. Main menu

Then another window appears "Education" see. Fig.3.

In this window, too, blank image makes it clear that the program has not started.

However, when clicking on the "Start" button is activated and the camera automatically detects a face in the image (photograph). If the image is missing, and the detector too. However, the camera is still running, waiting for the image.

To display the camera and detection used Emgu CV, which is a cross - platform .Net complement to the Open CV library for image processing.

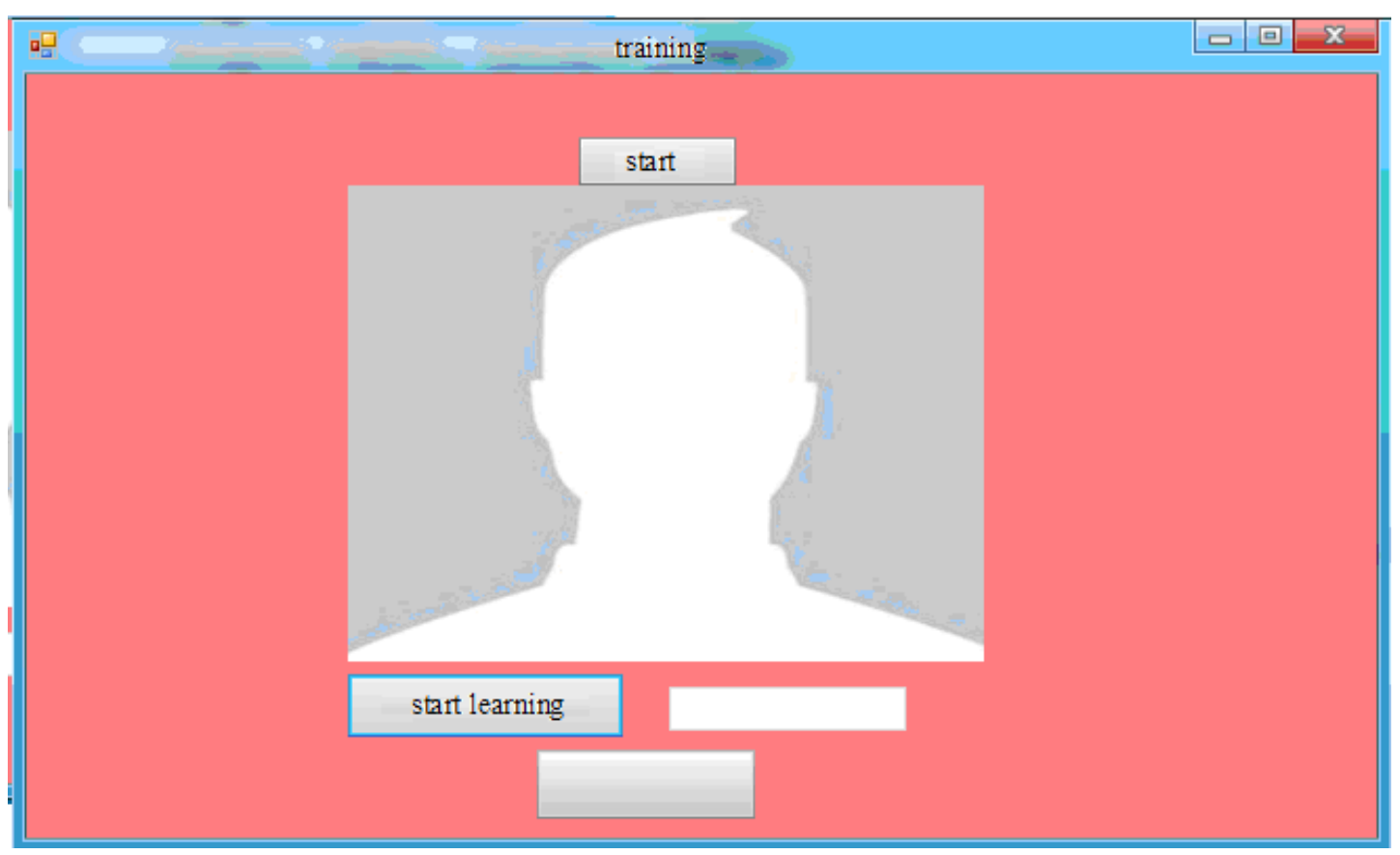

Fig. 3. Education

Finally, when the image of the image (photographs) of a person appears, the camera automatically recorded face of the person. Then at the bottom you need to write down the name and surname of the person. Then click on the "Start Training" button. 




Fig.4. Filling

Click on the "Start Training", you enable the program code that will cut out only the face, then translates it into a gray, converts it into a binary matrix and saves them into a specified folder. In this photo and name, and the file name of the matrix will be identical.

Fig. 4. it is clear that when you click on the "Start Training" button inscribed photo cut out with the transformation of gray, and then comes the recording process and displays a message that the person is recorded in the database. Next window "Education" closes and opens a new window for re-training of the next image, see. Fig.3. And so it goes, as long as you do not close the window independently. After that you need to close the main menu, see. Fig.2. Then run the program again.

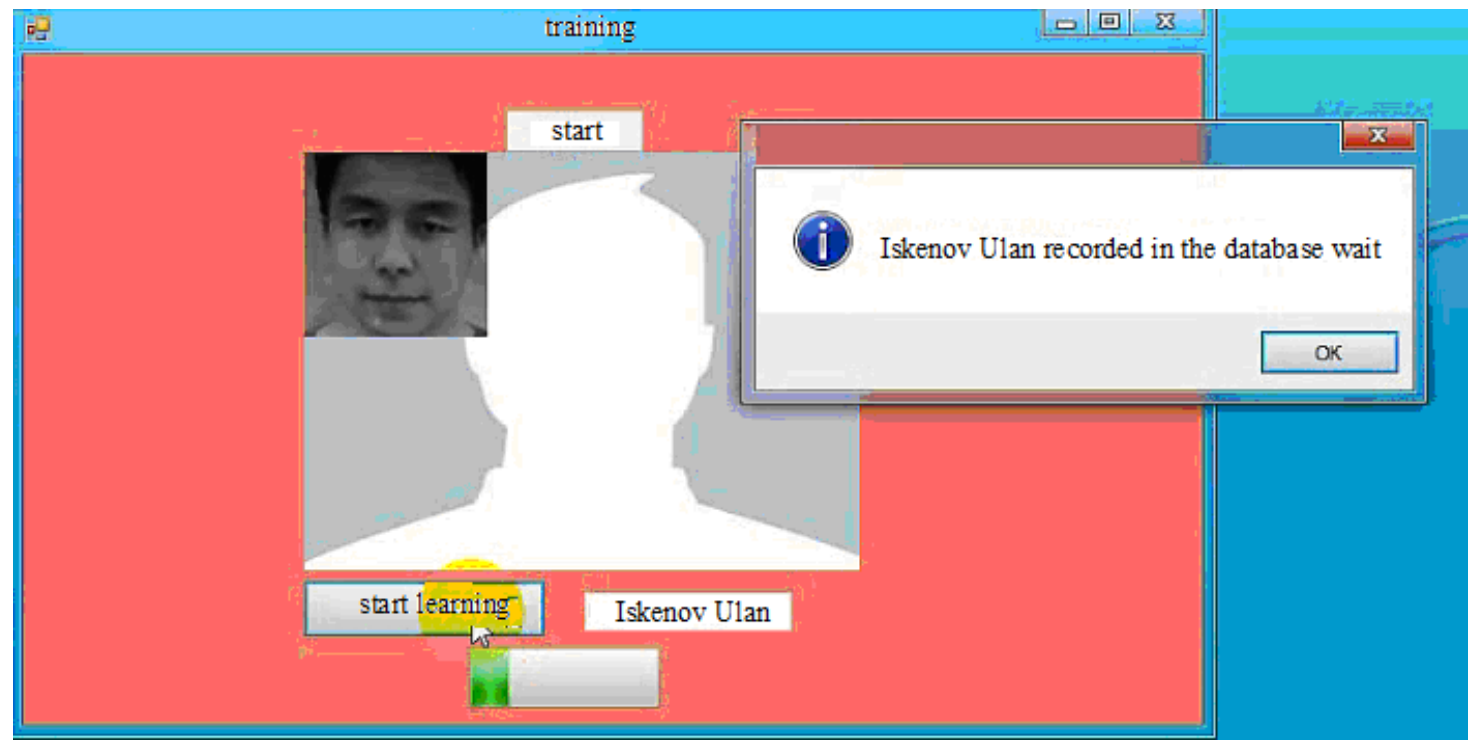

Fig.5. Completion of training

The recognition process

On the main menu, see. Fig.2. click on the "recognition" thereby activate the camera and the whole recognition process. 




Fig. 6. Recognition

Once the original camera finds the best matching standard, it displays the name of a reference person as a result. In addition, the name is displayed inscription indicating, that, for the base picture has. Otherwise, if you specify a person who has not yet been entered into the database name is not displayed and will indicate that "there are no Photography in", as shown in Fig. 7.



Fig. 7. Recognition 


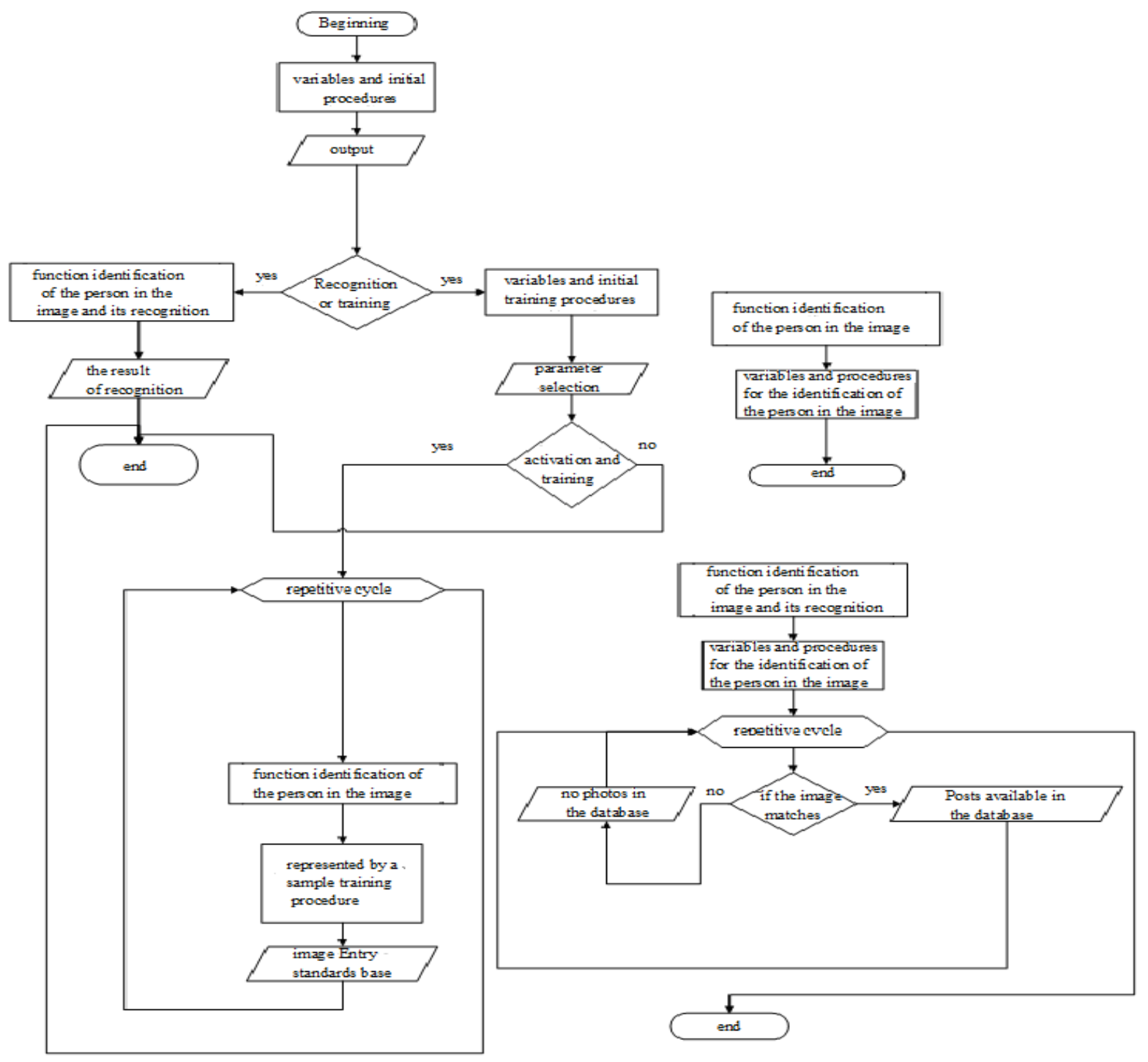

\section{CONCLUSIONS}

1. An algorithm for recognizing the structure of the device with the procedure training and the use of standards.

2. Resolves a specific task to photograph recognition

3. For a more accurate reliable work recognizes the device it is necessary to increase the number of cells the receptor matrix, as well as increase the base master images of recognizable objects.

\section{LITERATURE}

[1]. Hunt E. Artificial Intelligence. - M .: Mir, 1978

[2]. Simon Haykin. Neural networks: a complete course = Neural Networks: A Comprehensive Foundation. 2 nd ed. - M .: "Williams", 2006

[3]. Zhuravlev Y.I. Selected scientific papers. - Ed. Masters 2002 - 420C.

[4]. Batyrkanov J.I. Artificial Intelligence Systems. - B .: IC "Technique" 2013

[5]. Herbert Schildt "C \# 3.0, 4.0. The Complete Guide" 